\title{
Augmented Reality Based Product Invisible Area Assembly Assistance
}

\author{
Zenglei Wang, Shusheng Zhang* and Xiaoliang Bai \\ The Key Laboratory of Contemporary Designing and Integrated Manufacturing Technology, Ministry of Education, Northwestern \\ Polytechnical University, Xi’an, 710072, China \\ ${ }^{*}$ Corresponding author
}

\begin{abstract}
Aiming at the problem of the invisible area in a manual assembly process, this paper proposes an invisible area assembly scheme based on augmented reality technology. Different location tracking methods are put forward according to whether the objects to be assembled are visible. At the same time, the enhanced visual visualization technology of invisible area data is studied, so that invisible area information can be displayed to operators better. Finally, invisible area assembly experiments based on augmented reality technology were carried out. Experimental results show that this method can significantly improve the efficiency of assembly work in complex invisible areas and reduce the assembly error rate effectively.
\end{abstract}

Keywords-augmented reality; invisible area; assembly; efficiency; error rate

\section{INTRODUCTION}

Assembly is an integral part of the product manufacturing process, and its intelligence is often of poor degree. In the manual assembly process, workers often need to adjust the posture to see the assembly position information because of the complicated part structure. Even if it is adjusted anyway, there are always some necessary assembly visual information that is invisible, that is, the workers encounter assembly blind spots. The presence of blind spots in the assembly makes workers vulnerable to fatigue, and the assembly efficiency and accuracy are difficult to guarantee.

In view of the above problems, at present, the method of virtual simulation in the design stage $[1,2]$ is mainly used to construct the visible cone of the human eye and the visible field of the assembly part, and the accessibility of the assembled parts in the operator's field of view is evaluated. Visual accessibility is divided into four categories: fully visible, basically visible, barely visible, and invisible. For invisible situations, the product must return and modify the design or re-adjust the assembly plan. Although this method is simple and effective, it obviously increases the production cycle and the production cost.

In recent years, with the development of augmented reality technology, which provides a new solution for the assembly of blind spots in products. In this paper, the invisible area problem encountered in manual assembly is solved using the augmented reality technology, which improves the assembly efficiency and reduces the error rate and relieves the physical and mental fatigue of workers.

\section{TECHNICAL SOLUTIONS}

A. Overview

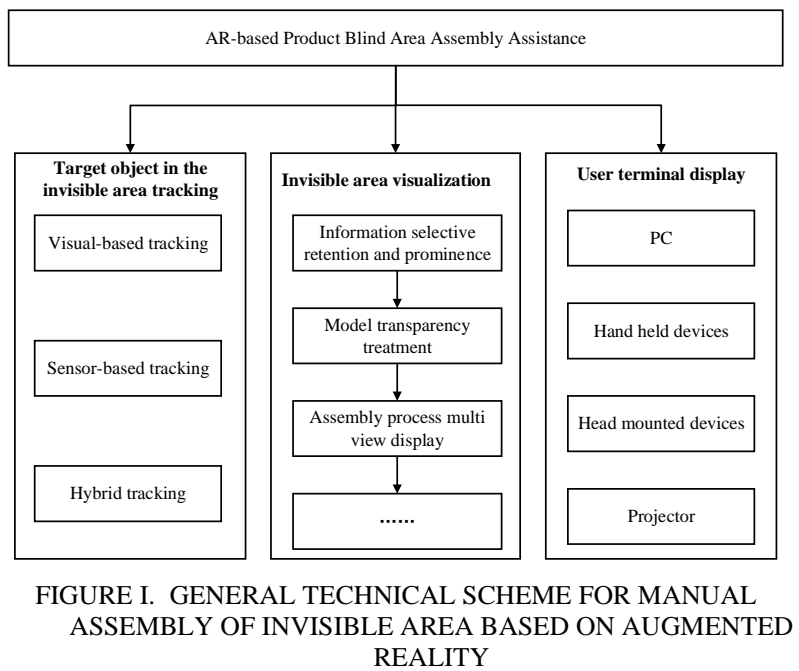

The manual assembly in invisible area based on augmented reality technology mainly includes three aspects: 1) object position in the invisible area tracking, 2) invisible area information visualization, and 3) user terminal display. As shown in Figure I, the tracking technology is used to capture the position and location of the target object in real time, and the captured position information is combined with the digitized model of the invisible area component or the human hand, and superimposed on the assembly scene by a certain visualization means on the terminal device. The invisible area information is presented to the workers in a visual, intuitive and natural way, thereby the problem of insufficient visual information in key parts of the invisible area assembly is solved.

\section{B. Invisible Area Object Tracking}

The most important thing in manual assembly is to track the target objects to be assembled in the invisible area. It is the key to realizing the correct superposition of virtual information. As seen in Figure II, $O_{w}-X_{w} Y_{w} Z_{w}$ is the world coordinate system, $O_{r}-X_{r} Y_{r} Z_{r}$ is the local coordinate system of the target object. $I=\overrightarrow{O_{w} O_{r}}$ represents a vector from the origin of the world coordinate system to the origin of the local coordinate system 
of the target target object. Vector $K=\overrightarrow{O_{r} P}$ represents the point from the origin of the local coordinate system of the target object to any point on it. The position of the origin $O_{r}$ of the object coordinate system to be assembled in the world coordinate system which can be represented by $I$, and the position of the point $P$ in the local coordinate system of the target object can be represented by $K$.

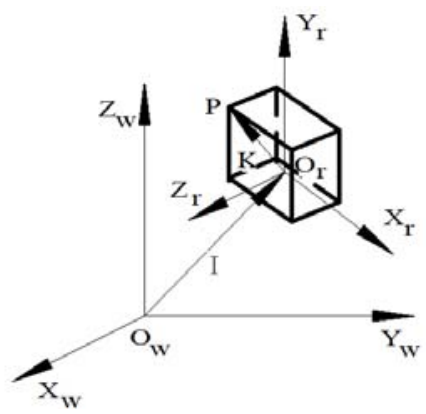

FIGURE II. DESCRIPTION OF THE POSE OF THE TARGET OBJECT

Assume $K_{x r}, K_{y r}$ and $K_{z r}$ represent the component on the xaxis, y-axis, and z-axis, respectively. Project $K_{x r}, K_{y r}$, and $K_{z r}$ to the world coordinate system, the coordinate of point $P$ can be obtained.

$$
\left\{\begin{array}{l}
K_{x}=K_{x r} l_{1}+K_{y r} l_{2}+K_{z r} l_{3}+I_{1} \\
K_{y}=K_{x r} m_{1}+K_{y r} m_{2}+K_{z r} m_{3}+I_{2} \\
K_{z}=K_{x r} n_{1}+K_{y r} n_{2}+K_{z r} n_{3}+I_{3}
\end{array}\right.
$$

Where $I_{1}, I_{2}$, and $I_{3}$ are the positional offsets of the local coordinate system origin on the coordinate axis of the world coordinate system $X, y, Z$, respectively. Use $<X_{w}, X_{r}>$ to indicate the angle between the axis unit vector $X_{w}$ and $X_{r}$. See Table 1:

TABLE I. COSINE TABLE OF THE ANGLE BETWEEN THE WORLD COORDINATE SYSTEM AND THE LOCAL COORDINATE SYSTEM

\begin{tabular}{cccc}
\hline \hline & $X_{r}$ & $Y_{r}$ & $Z_{r}$ \\
\hline \hline$X_{w}$ & $l_{1}=\cos <X_{w}, X_{r}>$ & $l_{2}=\cos <X_{w}, Y_{r}>$ & $l_{3}=\cos <X_{w}, Z_{r}>$ \\
$Y_{w}$ & $m_{1}=\cos <Y_{w}, X_{r}>$ & $m_{2}=\cos <Y_{w}, Y_{r}>$ & $m_{3}=\cos <Y_{w}, Z_{r}>$ \\
$Z_{w}$ & $n_{1}=\cos <Z_{w}, X_{r}>$ & $n_{2}=\cos <Z_{w}, Y_{r}>$ & $n_{3}=\cos <Z_{w}, Z_{r}>$ \\
\hline \hline
\end{tabular}

Assume the homogeneous coordinate of $P$ in the local coordinate system is $\left(x_{r}, y_{r}, z_{r}, 1\right)$, and the homogeneous coordinate in the world coordinate system is $\left(x_{w}, y_{w}, z_{w}, 1\right)$, then:

$$
\left\{\begin{array}{l}
x_{w}=x_{r} l_{1}+y_{r} l_{2}+z_{r} l_{3}+I_{1} \\
y_{w}=x_{r} m_{1}+x_{r} m_{2}+z_{r} m_{3}+I_{2} \\
z_{w}=x_{r} n_{1}+x_{r} n_{2}+z_{r} n_{3}+I_{3}
\end{array}\right.
$$

Which can be expressed with matrix:

$$
\left(\begin{array}{c}
x_{w} \\
y_{w} \\
z_{w} \\
1
\end{array}\right)=\left[\begin{array}{cccc}
l_{1} & l_{2} & l_{3} & I_{1} \\
m_{1} & m_{2} & m_{3} & I_{2} \\
n_{1} & n_{2} & n_{3} & I_{3} \\
0 & 0 & 0 & 1
\end{array}\right]\left(\begin{array}{c}
x_{r} \\
y_{r} \\
z_{r} \\
1
\end{array}\right)
$$

In a brief form:

$$
P_{w}=M P_{r}
$$

Where $M$ is a homogeneous transformation matrix, which can be used to describe the pose of the target object in the world coordinate system. $M$ can be decomposed into the following form:

$$
M=\left[\begin{array}{cccc}
l_{1} & l_{2} & l_{3} & I_{1} \\
m_{1} & m_{2} & m_{3} & I_{2} \\
n_{1} & n_{2} & n_{3} & I_{3} \\
0 & 0 & 0 & 1
\end{array}\right]=\left[\begin{array}{cccc}
1 & 0 & 0 & I_{1} \\
0 & 1 & 0 & I_{2} \\
0 & 0 & 1 & I_{3} \\
0 & 0 & 0 & 1
\end{array}\right]\left[\begin{array}{cccc}
l_{1} & l_{2} & l_{3} & 0 \\
m_{1} & m_{2} & m_{3} & 0 \\
n_{1} & n_{2} & n_{3} & 0 \\
0 & 0 & 0 & 1
\end{array}\right]=T R
$$

Where $T$ is the translation vector and $R$ is the rotation matrix. The translation vector $T$ can be seen as the local coordinate system of the target object parallel to the coordinate axis of the world coordinate system is moved, and $I_{1}, I_{2}, I_{3}$ represent the moving distance along the $X_{w}, Y_{w}, Z_{w}$ axes, respectively. The rotation matrix $R$ can be seen as the angle changes between the coordinate axes of the assembly object and the world coordinate, that is, only the rotation between the coordinate axes occurs. Thus, $T$ describes the position of the target object on the assembly, and R characterizes the posture of the target object on the assembly. Therefore, $M$ can be used to describe the spatial assembly pose of any target object in the assembly coordinate system.

\section{A. Tracking Method for the Target Object That Partially Visible}

For the case where the target object is partially visible, the structure exposed to the blind spot is tracked, thereby the entire target object can be tracked. The overall idea is to paste the artificial marker on the surface of the target object outside the invisible area, and track the target object by tracking the marker [6-8].

Taking tracking based on artificial markers as an example, as shown in Figure 3, in the real world, $M 1, M 2, M 3$ represents artificial markers attached to the plane $A, B, C$, respectively, and $M_{4}$ is an artificial marker attached to the object being tracked. Assume the shape of the object is a cube, and C1 represents a real camera for acquiring the entire scene. Through the camera calibration and the corresponding positioning and tracking algorithm, the pose of the camera relative to each artificial marker in the world coordinate system $O-X Y Z$ can be obtained. In the virtual world, the virtual camera C2 is used to 
render the model of each plane and the object being tracked from the same pose as the real camera. The dotted cube represents the theoretical pose $P^{\prime}$ of the tracked object, and the cube representation is tracked. The current pose $\mathrm{P}$ of the object model in the virtual world. In the virtual world, the homogeneous transformation matrix $\mathrm{Q}$ between the two can be obtained according to the current pose and the theoretical pose of the object being tracked.
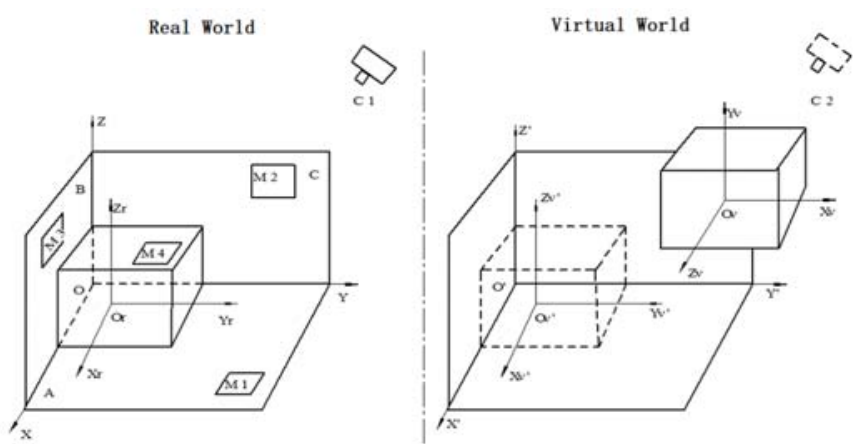

FIGURE III. PARTIALLY VISIBLE OBJECT TRACKING BASED ON ARTIFICIAL MARKERS

\section{B. Tracking Method for the Target Object That Invisible}

For the case where the target object is completely invisible, the pose of the target object can be indirectly estimated by tracking the human hand. As shown in Figure IV. , the coordinate system of the human hand is $O_{h}-X_{h} Y_{h} Z_{h}$, and the coordinate system of the target object is $O_{r}-X_{r} Y_{r} Z_{r}$. The essence of the positioning of the target object in the second type of invisible area is to require the coordinate system of the target object to be aligned with the coordinate system of the human hand. Sub-transform matrix $M$.

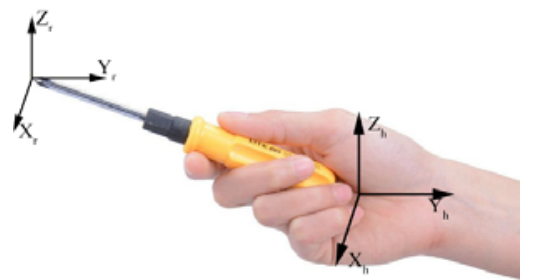

FIGURE IV. INVISIBLE TARGET OBJECT TRACKING BASED ON LEAP MOTION

In this paper, Leap Motion is used to obtain the real-time pose of the human hand in the sensor coordinate system [12]. The schematic diagram of Leap Motion is shown in Figure 5. The pose of the target object is determined according to the posture of the human hand and the relative positional relationship between the human hand and the target object.
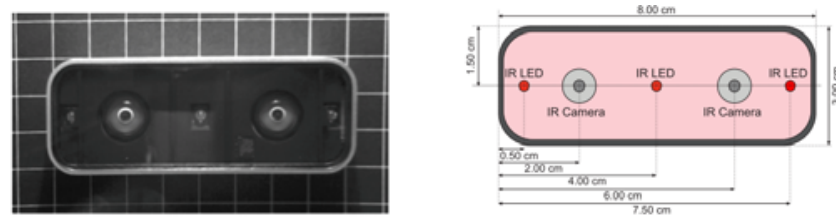

FIGURE V. THE SCHEMATIC DIAGRAM OF LEAP MOTION

\section{Invisible Area Information Visualization Enhancement}

After acquiring the position and posture of the invisible area object, by superimposing the corresponding three-dimensional geometric model on the surface of the assembly, the invisible area information visualization processing in the augmented reality environment (for example, superimposing the threedimensional model and selectively expressing, transparent processing, etc.) is realized. On one hand, it can enable workers to see the assembly parts in the model, so that the blind spot information changes from "invisible" to "visible". On the other hand, the main information details affecting the assembly in the invisible area can be highlighted in the workers. As shown in Figure VI, (a) is a three-dimensional geometric model of the box in the virtual environment, and the left inner wall has a plurality of screw-like schematic models, but the internal information of the box is blocked by a white obstacle model. The person cannot know the screw installation position information of the inside of the box from the outside of the box. In a real blind spot assembly scenario, such a white obstacle may be part of the structure of the assembly itself, or it may be a human hand and a hand-held component or tool during assembly, abstracting it into a white planar model. In the following, according to different situations, the display technology of the invisible area obstacle model and the invisible area assembly site object model to be assembled is discussed.
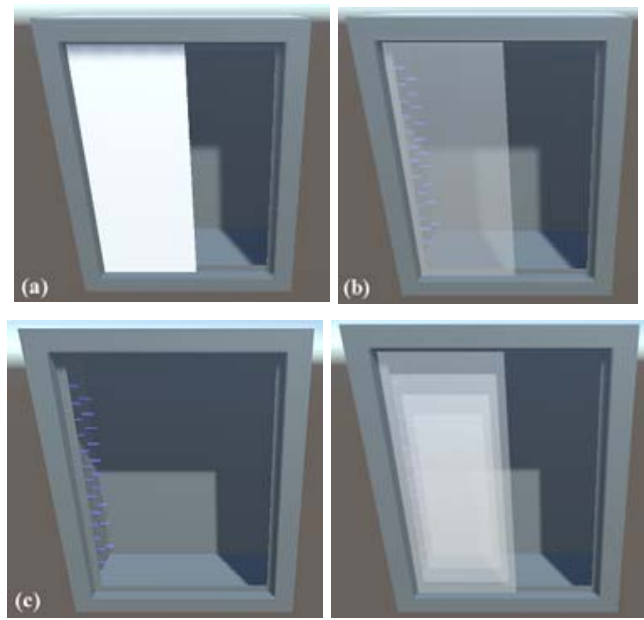

FIGURE VI. BLIND AREA OBSTACLE AND OBJECT TO BE ASSEMBLED MODEL PROCESSING (A) OUTWARD APPEARANCE OF BLIND AREA, WHITE OBSTACLE MODEL (B) OBSTACLE MODEL TRANSPARENCY PROCESSING, (C) REMOVAL OF OBSTACLE MODEL

When the white obstacle is part of the assembly, we cover the assembly model and the transparent white obstacle model onto the real assembly, so that the workers have the same ability like the perspective eye, they can see the internal information of the model directly through the obstacle. However, when there are more than one such obstacles and overlap each other, visual interference is caused by the transparency processing, as shown in (d). At this time, it is considered to remove some or all of the obstacle models for assembly. (c) shows the schematic of the obstacle removal model. Removing all obstacle models can visually see invisible area information, but losing the authenticity of the invisible area structure may lead to workers' 
misoperations. For example, in the case shown in (c), workers may mistakenly think that there are no obstacles existence, they may directly extend the hand from the position where the obstacle exists to the blind spot, causing collision between the human hand and the obstacle. If the obstacle is a relatively sharp component, the worker may be injured.

For the model display of the assembly site, we highlight the color and display the different angles and degrees of information in the virtual space multi-view rendering by changing the color. As shown in Figure VII, it is assumed that it is necessary to assemble in the red part of the figure. Since the color is red and the other parts are light blue, the worker can easily distinguish it from other parts, so that attention can be paid to the assembly. In addition, the plane on the right side of the box in the figure shows the viewing angle of the assembly part from the inside of the box and the upper part of the red assembly. Through such multi-view expression, the worker can visually obtain more information about the assembly part.

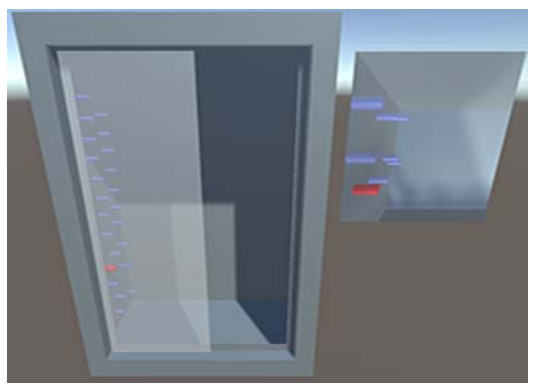

FIGURE VII. DISPLAY AND MULTI VIEW EXPRESSION OF ASSEMBLY AREA IN BLIND AREA

\section{RESULTS AND DISCUSSIONS}

\section{A. System Hardware and Software Setup}

In order to integrate the two invisible situations, we designed an invisible area assembly hardware system. As shown in Figure VIII, a connector 1 manufactured by 3D printing technology is attached to the back of the cabinet, and the connector 2 is fixed on the table. The connectors of the two cooperated with each other. The HTC Vive handles are used to track the two connectors separately, and the Vive helmet is used to display augmented reality scenes and assembly models. In the process of manually manipulating the box to dock the two connectors together, since the shape of the body is bulky, the sight line of the human eye is blocked to see the connector.

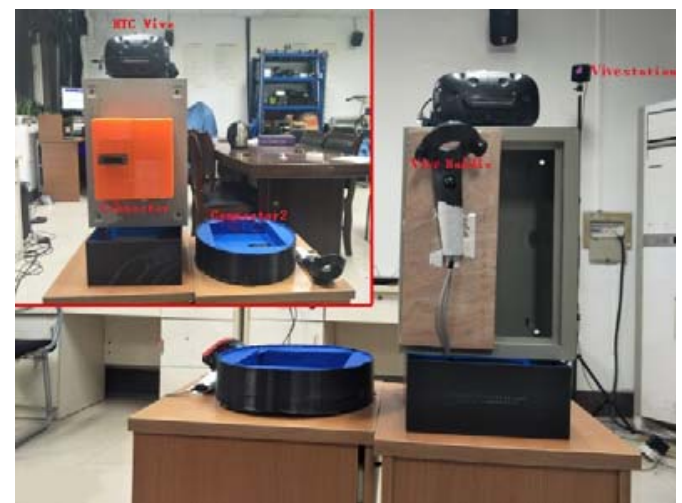

FIGURE VIII. SYSTEM HARDWARE SETUP

Meanwhile, due to the existence of a wooden board on the front side of the cabinet, the internal information is not easily seen from the outside. There are 20 screws on the side wall of the cabinet, and the distribution is shown in Figure IX. (right). When a person carries a component into the interior of the cabinet and moves toward the side wall, the person cannot obtain information of the human hand and the object in the hand, and thus the target object is invisible. We use a Leap Motion attached to the inner surface of the top cabinet to track the person hand entering the blind spot (see Figure IX. (left)).
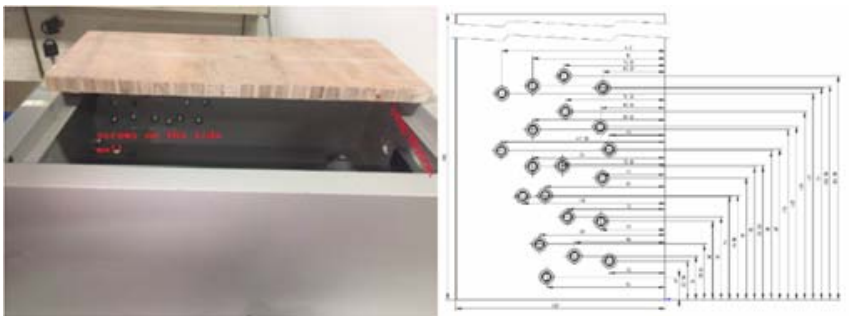

FIGURE IX. CABINET (LEFT) AND SCREWS DISTRIBUTION (RIGHT)

HTC Vive is used as the user terminal display device, and Unity3D is used as software development environment, Steam VR is used to realize the pose tracking of Vive helmet, handle and Tracker.

\section{B. Experimental Results}

In the first type of invisible area assembly, as shown in Figure X. (a), the participant wears HTC Vive and sees the real world through the video captured by Vive's embedded front camera. Two connector models are superimposed on the video stream. After the experiment started, the operator picked up the box from the desktop, the plug model was seen through the model of the transparent connector 1 superimposed on the video stream, and the plug portion of the plug was adjusted. The relative position of the insertion portion of the jack is inserted into the base. Compared with the case without augmented reality, the operation efficiency is greatly improved. 


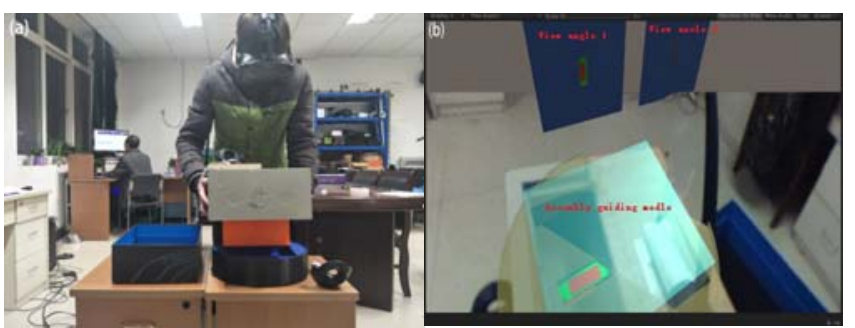

FIGURE X. PARTIALLY VISIBLE PART ASSEMBLY BASED ON AUGMENTED REALITY

In the second type of invisible area assembly, as shown in Figure XI, the participant wears HTC Vive and sees the human hand, the box and the screw model on the inner wall of the box. The target screw color is different from other screws. The screw was held by the hand, as shown in Figure XI. (b). When the experiment starts, the participant will put the hand into the invisible area. According to the hand model detected by Leap Motion, adjust the relative position of the target screw on the inside of the hand and the inside of the box, and install the nut to corresponding screws. Assembly efficiency and assembly accuracy are significantly improved compared to the case without augmented reality assistance.

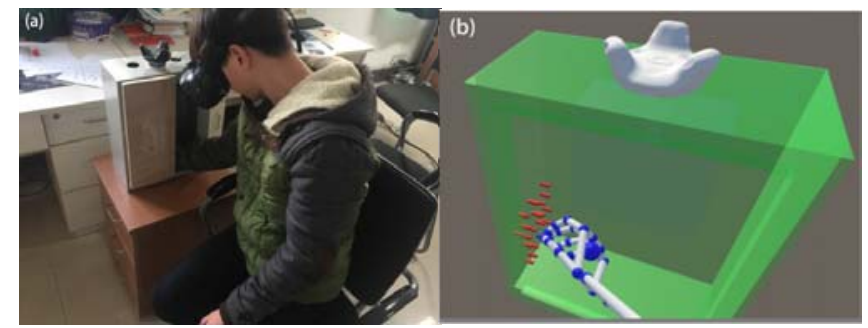

FIGURE XI. INVISIBLE PART ASSEMBLY BASED ON AUGMENTED

$$
\text { REALITY }
$$

\section{CONCLUSION}

In this paper, the invisible area operation problem in manual assembly is analyzed. According to different invisible area conditions, a universal augmented reality solution is given. Through the augmented reality technology, the threedimensional model of the assembly part, the human hand and the hand-held parts or tools in the blind zone is superimposed on the real scene, and then the transparent model display processing, model surface reduction and color highlighting are performed, so that the blind zone information is visualized to the manipulator. The experimental results show that the proposed method can significantly improve the efficiency of assembly operations in complex invisible areas and effectively reduce the assembly error rate.

\section{REFERENCES}

[1] Kang Y. Research on Quantitative Calculation of Visual Factors in Virtual Assembly System [D]. Xidian University, 2015.

[2] Zeng Y. Research on Analysis and Evaluation of Reachability Based on Maintainability Design[D]. National University of Defense Technology,2017.

[3] Henderson S, Feiner S. Exploring the Benefits of Augmented Reality Documentation for Maintenance and Repair[J]. IEEE Transactions on Visualization \& Computer Graphics, 2011, 17(10):1355.
[4] Wang Y, Zhang S, Yang S, et al. P oint Cloud and Visual Feature-Based Tracking Method for an Augmented Reality-Aided Mechanical Assembly System [J]. International Journal of Advanced Manufacturing Technology, 2018.

[5] Wang Y, Zhang S, Yang S, et al. A LINE-MOD-based markerless tracking approachfor AR applications[J]. International Journal of Advanced Manufacturing Technology, 2017, 89(5-8):1699-1707.

[6] Westerfield G, Mitrovic A, Billinghurst M. Intelligent Augmented Reality Training for Motherboard Assembly[J]. International Journal of Artificial Intelligence in Education, 2015, 25(1):157-172.

[7] Fiorentino M, Uva A E, Gattullo M, et al. Augmented reality on large screen for interactive maintenance instructions[J]. Computers in Industry, 2014, 65(2):270-278.

[8] Hou L, Wang X, Truijens M. Using Augmented Reality to Facilitate Piping Assembly: An Experiment-Based Evaluation[J]. Journal of Computing in Civil Engineering, 2012, 29(1):05014007.

[9] Syberfeldt A, Danielsson O, Holm M, et al. Augmented Reality at the Industrial Shop-Floor[M]// Augmented and Virtual Reality. Springer International Publishing, 2014:201-209.

[10] Crivellaro A, Rad M, Verdie Y, et al. A Novel Representation of Parts for Accurate 3D Object Detection and Tracking in Monocular Images[C]// IEEE International Conference on Computer Vision. IEEE, 2015:43914399.

[11] Rodrigues J J, Kim J S, Furukawa M, et al. 6D pose estimation of textureless shiny objects using random ferns for bin-picking[C]// Ieee/rsj International Conference on Intelligent Robots and Systems. IEEE, 2012:3334-3341.

[12] Leap, Developer[EB/OL]. http://developer.leapmotion.com/, 2015. 